\title{
Information Stability in Heat-Assisted Magnetic Recording
}

\author{
T. Kobayashi, Y. Nakatani*, and Y. Fujiwara \\ Graduate School of Engineering, Mie Univ., 1577 Kurimamachiya-cho, Tsu 514-8507, Japan \\ *Graduate School of Informatics and Engineering, Univ. of Electro-Communications, 1-5-1 Chofugaoka, Chofu 182-8585, Japan
}

The thermal stability factor $K_{\mathrm{um}} V_{\mathrm{m}} / k T$ and the anisotropy constant ratio $K_{\mathrm{u}} / K_{\text {bulk }}$ necessary for 10 years of archiving in heat-assisted magnetic recording of 2 Tbpsi are evaluated by employing a bit error rate calculation using a grain error probability $P$. Although the attempt frequency $f_{0}$ in $P$ is a function of the Gilbert damping constant, the Curie temperature, $K_{\mathrm{u}} / K_{\text {bulk }}$, the grain volume, and temperature, $f_{0}$ can be treated as a constant. The Gilbert damping constant and the Curie temperature variation are parameters with little impact. On the other hand, the grain size variation, the grain number per bit $n$, the mean Curie temperature $T_{\mathrm{cm}}$, and the storage temperature $T$ are parameters with a strong impact on bit error rate. Although $K_{\mathrm{um}} V_{\mathrm{m}} / k T$ decreases as $n$ increases due to a statistical problem, a larger $K_{\mathrm{u}} / K_{\text {bulk }}$ is necessary as $n$ increases due to a smaller grain size. A larger $K_{\mathrm{u}} / K_{\text {bulk }}$ is also necessary as $T_{\mathrm{cm}}$ decreases. The bit error rate increases rapidly as $T$ increases.

Key words: thermal stability factor, anisotropy constant ratio, bit error rate, probability, attempt frequency

\section{Introduction}

The long-term stability of archived information is one of the most important properties of magnetic recording media. This stability has been widely discussed using the thermal stability factor $K_{\mathrm{um}} V_{\mathrm{m}} / k T$, where $K_{\mathrm{um}}$, $V_{\mathrm{m}}, k$, and $T$ are the mean grain anisotropy constant, the grain volume for the mean grain size of the medium, the Boltzmann constant, and temperature, respectively ${ }^{1)}$. We have already estimated the numerical value of $K_{\mathrm{um}} V_{\mathrm{m}} / k T$ by employing a bit error rate calculation using the grain error probability for various grain numbers per bit $n$ and the standard deviations of grain size $\sigma_{\mathrm{D}}{ }^{2), 3)}$. In that calculation, we used a constant attempt frequency $f_{0}$ of $1.0 \times 10^{11} \mathrm{~s}^{-1}$. However, precisely speaking, $f_{0}$ is a function of the Gilbert damping constant $\alpha$, the magnetization $M_{\mathrm{s}}$, the anisotropy field $H_{\mathrm{k}}$, the grain volume $V$, and $T^{4}$.

Heat-assisted magnetic recording (HAMR) is a promising candidate as a next generation magnetic recording method that can operate beyond the trilemma limit ${ }^{1}$. HAMR is a recording method in which the medium is heated to reduce coercivity during the writing period. We have introduced a HAMR design parameter, namely, the medium anisotropy constant ratio $K_{\mathrm{u}} / K_{\text {bulk }}$ 5) in place of the medium anisotropy constant $K_{\mathrm{u}}$ since the $K_{\mathrm{u}}$ value at the storage temperature is a function of the medium Curie temperature $T_{\mathrm{c}}$, which is strongly related to the writing property. $K_{\mathrm{u}} / K_{\text {bulk }}$ is the intrinsic ratio of the medium $K_{\mathrm{u}}$ to bulk FePt $K_{\mathrm{u}}$ regardless of $T_{\mathrm{c}}$ where FePt is a candidate HAMR medium material thanks to its large $K_{\mathrm{u}}$ and a relatively low $T_{\mathrm{c}}$. A medium with a high $K_{\mathrm{u}} / K_{\text {bulk }}, \quad$ e.g. $0.6-1.0^{6)}$, is difficult to manufacture regardless of $T_{\mathrm{c}} . M_{\mathrm{s}}$ is a function of $T_{\mathrm{c}}$ and $T . H_{\mathrm{k}}=2 K_{\mathrm{u}} / M_{\mathrm{s}}$ is a function of $T_{\mathrm{c}}, K_{\mathrm{u}} / K_{\text {bulk }}$, and $T$. And $V_{\mathrm{m}}$ is a function of $n$ under a constant grain height. Therefore, $f_{0}$ is a function of $\alpha, T_{\mathrm{c}}, T$, $K_{\mathrm{u}} / K_{\text {bulk }}$, and $n$.

In this paper, we evaluate the $K_{\mathrm{um}} V_{\mathrm{m}} / k T$ and $K_{\mathrm{u}} / K_{\text {bulk }}$ necessary for 10 years of archiving in 2 Tbpsi HAMR by employing a bit error rate calculation using the grain error probability, which includes $f_{0}$ as a function of $\alpha, T_{\mathrm{c}}, T, K_{\mathrm{u}} / K_{\text {bulk }}$, and $n$. Furthermore, we also examine the dependence of the bit error rate on the standard deviation of the Curie temperature as well as that of the grain size.

\section{Calculation Method}

\subsection{Attempt frequency}

The thermal stability factor $K_{\mathrm{um}} V_{\mathrm{m}} / k T$ and the anisotropy constant ratio $K_{\mathrm{u}} / K_{\text {bulk }}$ necessary for 10 years of archiving in 2 Tbpsi HAMR were evaluated by employing a bit error rate calculation using the grain error probability $P$

$$
P=1-\exp \left(-f_{0} t \exp \left(-\frac{K_{\mathrm{u}} V_{\mathrm{m}}}{k T} \cdot\left(\frac{D}{D_{\mathrm{m}}}\right)^{2}\right)\right)
$$

where $f_{0}, t, K_{\mathrm{u}}, V_{\mathrm{m}}, k, T$, and $D$ are the attempt frequency, time, the grain anisotropy constant, the grain volume for mean grain size $D_{\mathrm{m}}$, the Boltzmann constant, temperature, and the grain size, respectively.

The attempt frequency $f_{0}$ has been deduced as

$f_{0}=\frac{\alpha \gamma}{1+\alpha^{2}} \sqrt{\frac{M_{\mathrm{s}} H_{\mathrm{k}}^{3} V}{2 \pi k T}}\left(1-\left(\frac{H}{H_{\mathrm{k}}}\right)^{2}\right)\left(1+\frac{H}{H_{\mathrm{k}}}\right)$

under a magnetic field $H$ where $\alpha, \gamma, M_{\mathrm{s}}, H_{\mathrm{k}}$, and $V$ are the Gilbert damping constant, the gyromagnetic ratio, the magnetization, the anisotropy field $H_{\mathrm{k}}=2 K_{\mathrm{u}} / M_{\mathrm{s}}$, and the grain volume, respectively ${ }^{4}$. $M_{\mathrm{s}}$ and $H$ change from parallel to antiparallel for $H$ $>0$, and antiparallel to parallel for $H<0$. 


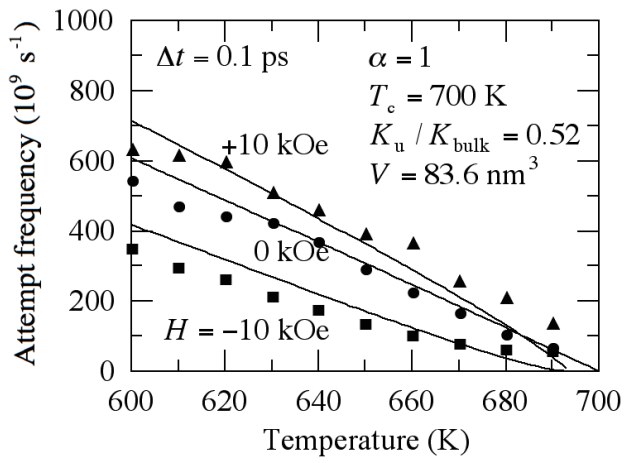

Fig. 1 Temperature dependence of attempt frequency for various magnetic fields $H$.

Figure 1 shows the temperature dependence of $f_{0}$ for various $H$ values under the conditions where $\alpha=$ 1 , the Curie temperature $T_{\mathrm{c}}=700 \mathrm{~K}, K_{\mathrm{u}} / K_{\text {bulk }}=0.52$, and $V=83.6 \mathrm{~nm}^{3}$.

The solid lines were calculated using Eq. (2). The temperature dependence of $M_{\mathrm{s}}$ was determined by employing a mean field analysis ${ }^{7}$, and that of $K_{\mathrm{u}}$ was assumed to be proportional to $M_{\mathrm{s}}^{28}$ ) . The $T_{\mathrm{c}}$ value was adjusted by the $\mathrm{Cu}$ simple dilution of $\left(\mathrm{Fe}_{0.5} \mathrm{Pt}_{0.5}\right)_{1-z} \mathrm{Cu}_{z}$. $M_{\mathrm{s}}\left(T_{\mathrm{c}}, T\right)$ is a function of $T_{\mathrm{c}}$ and $T$, and $M_{\mathrm{s}}\left(T_{\mathrm{c}}=770 \mathrm{~K}, T=300 \mathrm{~K}\right)=1000 \mathrm{emu} / \mathrm{cm}^{3}$ was assumed for $\mathrm{FePt}(\mathrm{Cu}$ composition $z=0)$. $K_{\mathrm{u}}\left(T_{\mathrm{c}}\right.$, $\left.K_{\mathrm{u}} / K_{\text {bulk }}, T\right)$ is a function of $T_{\mathrm{c}}, K_{\mathrm{u}} / K_{\text {bulk }}$, and $T$ where

$$
K_{\mathrm{u}} / K_{\text {bulk }}=\frac{K_{\mathrm{u}}\left(T_{\mathrm{c}}, K_{\mathrm{u}} / K_{\text {bulk }}, T\right)}{K_{\mathrm{u}}\left(T_{\mathrm{c}}, K_{\mathrm{u}} / K_{\text {bulk }}=1, T\right)} .
$$

$K_{\mathrm{u}}\left(T_{\mathrm{c}}=770 \mathrm{~K}, K_{\mathrm{u}} / K_{\text {bulk }}=1, T=300 \mathrm{~K}\right)$ $\mathrm{Merg} / \mathrm{cm}^{3}$ was assumed for $\mathrm{FePt}(z=0)$. We used $M_{\mathrm{s}}\left(T_{\mathrm{c}}=700 \mathrm{~K}, T\right) \quad$ and $\quad K_{\mathrm{u}}\left(T_{\mathrm{c}}=700 \mathrm{~K}, K_{\mathrm{u}} / K_{\text {bulk }}\right.$ $=0.52, T)$ in Fig. 1 .

The filled symbols were obtained with a conventional micromagnetic calculation using the Landau-LifshitzGilbert equation ${ }^{9}$. The calculation step time $\Delta t$ was 0.1 ps. Usually Landau-Lifshitz-Bloch (LLB) equation is used for the simulation near Curie temperature ${ }^{10}$. However we used the Langevin equation with the effect of the temperature on the material parameters, which is the biggest different point between the LLB and the Langevin equation. Therefore the effect of the equation on the simulation is permissible.

Although we discuss the information stability around room temperature in this paper, the comparison was carried out for high temperature region since room temperature is too low to calculate in a short time when employing the micromagnetic calculation. Since the results represented by the solid lines and the filled symbols agree well, the attempt frequency was calculated using Eq. (2) in this paper.

Neither the demagnetizing nor the magnetostatic fields were considered in this paper for simplifying the model calculation. The problem of these fields is a future subject.

\subsection{Bit error rate calculation}

The bit error rate was calculated using each grain error probability $P_{i}$ of Eq. (1). For example, for 4 grains/bit, the 1 grain-error bit error rate ${ }_{4} b R_{1}$ is expressed as

$$
\begin{aligned}
& { }_{4} \mathrm{bER}_{1}=\operatorname{Er}_{1} P_{1}\left(1-P_{2}\right)\left(1-P_{3}\right)\left(1-P_{4}\right)+\cdots \\
& +\operatorname{Er}_{4}\left(1-P_{1}\right)\left(1-P_{2}\right)\left(1-P_{3}\right) P_{4}
\end{aligned},
$$

the 2 grain-error bit error rate ${ }_{4} \mathrm{bER}_{2}$

$$
\begin{aligned}
& { }_{4} \mathrm{bER}_{2}=\operatorname{Er}_{12} P_{1} P_{2}\left(1-P_{3}\right)\left(1-P_{4}\right)+\cdots \\
& +E r_{34}\left(1-P_{1}\right)\left(1-P_{2}\right) P_{3} P_{4}
\end{aligned},
$$

the 3 grain-error bit error rate ${ }_{4} \mathrm{bER}_{3}$

$$
\begin{aligned}
& { }_{4} \mathrm{bER}_{3}=E r_{123} P_{1} P_{2} P_{3}\left(1-P_{4}\right)+\cdots \\
& +E r_{234}\left(1-P_{1}\right) P_{2} P_{3} P_{4}
\end{aligned},
$$

and the 4 grain-error bit error rate ${ }_{4} \mathrm{bER}_{4}$

$$
{ }_{4} \mathrm{bER}_{4}=P_{1} P_{2} P_{3} P_{4}
$$

where

$$
E r_{j}=1 \text {, if } \frac{\sum_{i} M_{\mathrm{si}}\left(T_{\mathrm{c} i}, T\right) D_{i}^{2}}{n \cdot M_{\mathrm{s}}\left(T_{\mathrm{cm}}, T\right) D_{\mathrm{m}}^{2}}<0.5,
$$

and

$$
E r_{j}=0 \text {, if } \frac{\sum_{i} M_{\mathrm{si}}\left(T_{\mathrm{c} i}, T\right) D_{i}^{2}}{n \cdot M_{\mathrm{s}}\left(T_{\mathrm{cm}}, T\right) D_{\mathrm{m}}^{2}} \geq 0.5 .
$$

Errors occur in some grains of a bit. We assume that if the surface magnetic charge of the grains where the magnetization turns in the recording direction $\sum M_{\mathrm{s} i}\left(T_{\mathrm{c} i}, T\right) D_{i}^{2}$ is more than $50 \%$ (signal threshold) of the total surface magnetic charge in a bit $n \cdot M_{\mathrm{s}}\left(T_{\mathrm{cm}}, T\right) D_{\mathrm{m}}^{2}$, the bit is error free where $M_{\mathrm{si}}, T_{\mathrm{c} i}$, $D_{i}, n, T_{\mathrm{cm}}$, and $D_{\mathrm{m}}$ are the magnetization, the Curie temperature, the grain size of the $i$-th grain, the grain number per bit, the mean Curie temperature, and the mean grain size, respectively.

After all, the total bit error rate ${ }_{4} \mathrm{bER}$ is the summation of each bit error rate ${ }_{4} \mathrm{bER}_{k}$ as follows

$$
{ }_{4} \mathrm{bER}=\sum_{k}{ }_{4} \mathrm{bER}_{k} .
$$

The bit error rate is a function of the anisotropy constant ratio $K_{\mathrm{u}} / K_{\text {bulk }}$. Therefore, we obtained $K_{\mathrm{u}} / K_{\text {bulk }}$ for a certain bit error rate. Since the mean anisotropy constant $K_{\mathrm{um}}$ is a function of $K_{\mathrm{u}} / K_{\text {bulk }}$, the thermal stability factor $K_{\mathrm{um}} V_{\mathrm{m}} / k T$ was calculated using $K_{\mathrm{u}} / K_{\text {bulk }}$.

\section{Calculation Results}

\subsection{Information stability for 10 years of archiving}

The calculation conditions are summarized in Table 1 . The recording density is 2 Tbpsi, and the area $S$ of a bit is $323 \mathrm{~nm}^{2}$. The medium was assumed to be 
granular in which each bit has $n$ grains/bit. The mean grain size $D_{\mathrm{m}}$ was determined by

$$
D_{\mathrm{m}}=\sqrt{\frac{S}{n}}-\Delta
$$

where the non-magnetic spacing $\Delta$ was assumed to be $1 \mathrm{~nm}$. The grain height $h$ was $8 \mathrm{~nm}$, and so the grain volume $V_{\mathrm{m}}$ for $D_{\mathrm{m}}$ was $D_{\mathrm{m}} \times D_{\mathrm{m}} \times h$.

Table 1 Calculation conditions for 10 years of archiving.

\begin{tabular}{l|c}
\hline Recording density $($ Tbpsi) & 2 \\
Non - magnetic spacing $\Delta(\mathrm{nm})$ & 1 \\
Grain height $h(\mathrm{~nm})$ & 8 \\
Storage temperature $T(\mathrm{~K})$ & 300 \\
Storage time $t$ (years) & 10 \\
Signal threshold & 0.5 \\
Bit number (bits) & $10^{7}$ \\
Bit error rate & $1 \mathrm{E}-3$ \\
\hline
\end{tabular}

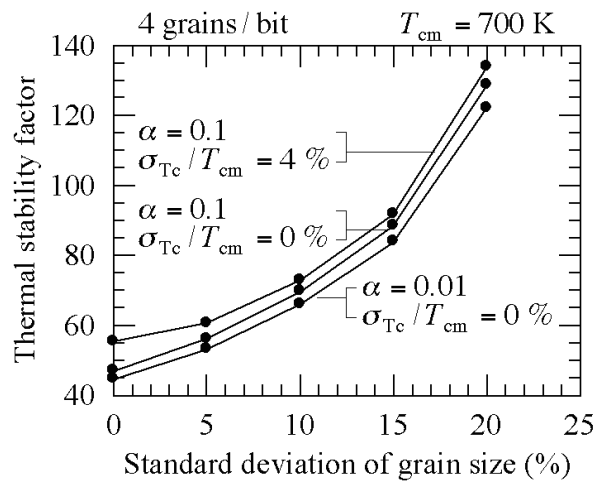

Fig. 2 Thermal stability factor required for 10 years of archiving as a function of the standard deviation of the grain size for various damping constants $\alpha$ and standard deviations of Curie temperature $\sigma_{\mathrm{Tc}} / T_{\mathrm{cm}}$.

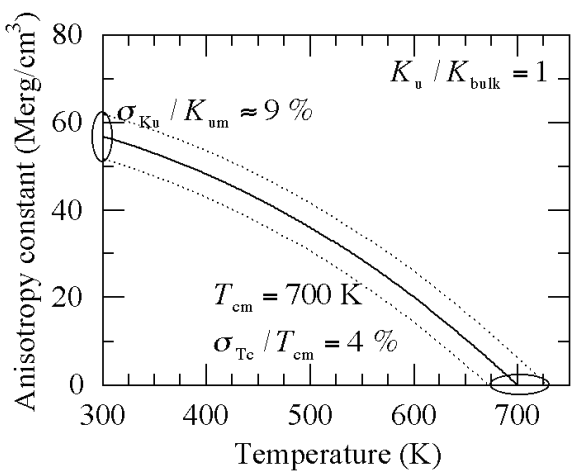

Fig. 3 Temperature dependence of anisotropy constant for standard deviation of Curie temperature $\sigma_{\mathrm{Tc}} / T_{\mathrm{cm}}=$ $4 \%$.

The storage temperature and the storage time were $300 \mathrm{~K}$ and 10 years, respectively. The signal threshold was 0.5 , and the calculation bit number was $10^{7}$. The thermal stability factor $K_{\mathrm{um}} V_{\mathrm{m}} / k T$ and the anisotropy constant ratio $K_{\mathrm{u}} / K_{\text {bulk }}$ were evaluated for a bit error rate of $1 \mathrm{E}-3$. We assumed that the grain size distribution was log-normal with a standard deviation of $\sigma_{\mathrm{D}}$, and that the Curie temperature distribution was normal with a mean Curie temperature and standard deviation of $T_{\mathrm{cm}}$ and $\sigma_{\mathrm{Tc}}$, respectively.

Figure 2 shows $K_{\mathrm{um}} V_{\mathrm{m}} / k T$ needed for 10 years of archiving as a function of $\sigma_{\mathrm{D}} / D_{\mathrm{m}}$ for various $\alpha$ and $\sigma_{\mathrm{Tc}} / T_{\mathrm{cm}}$ values under the conditions of 4 grains/bit and $T_{\mathrm{cm}}=700 \mathrm{~K}$. The $K_{\mathrm{um}} V_{\mathrm{m}} / k T$ value is strongly dependent on $\sigma_{\mathrm{D}} / D_{\mathrm{m}}$. However, $K_{\mathrm{um}} V_{\mathrm{m}} / k T$ is weakly dependent on $\alpha$ and $\sigma_{\mathrm{Tc}} / T_{\mathrm{cm}}$. When the error is low, Eq. (1) can be approximated as Eq. (12).

$$
P \approx f_{0} t \exp \left(-\frac{K_{\mathrm{u}} V_{\mathrm{m}}}{k T} \cdot\left(\frac{D}{D_{\mathrm{m}}}\right)^{2}\right) .
$$

The variables in an exponential function become parameters with a strong impact on bit error rate. Therefore, $\sigma_{\mathrm{D}} / D_{\mathrm{m}}$ has a strong effect.

On the other hand, $\alpha$ is only related to the attempt frequency $f_{0}$, and so a difference of even 10 times $\alpha$, namely $f_{0}$ has little effect on $K_{\mathrm{um}} V_{\mathrm{m}} / k T$ needed for 10 years of archiving as shown in Fig. 2 since $f_{0}$ is not an exponential variable. Therefore, $\alpha$ has only a weak effect, and some change in $f_{0}$ by changing $T_{\mathrm{c}}, T$, $K_{\mathrm{u}} / K_{\text {bulk }}$, or $n$ also has little effect on $K_{\mathrm{um}} V_{\mathrm{m}} / k T$. Therefore, $f_{0}$ can be treated as a constant in this paper. Furthermore, from Eq. (12), time $t$ as well as $f_{0}$ is a parameter with little impact on bit error rate.

The existence of $\sigma_{\mathrm{Tc}} / T_{\mathrm{cm}}=4 \%{ }^{11)}$ also has only a weak effect on the bit error rate as shown in Fig. 2. This is explained using the temperature dependence of $K_{\mathrm{u}}$ as shown in Fig. 3. When $\sigma_{\mathrm{Tc}} / T_{\mathrm{cm}}$ is $4 \%$, the standard deviation of the anisotropy constant $\sigma_{\mathrm{Ku}} / K_{\mathrm{um}}$ is $9 \%$ at $300 \mathrm{~K}$. The $\sigma_{\mathrm{Ku}} / K_{\mathrm{um}}$ value of $9 \%$ corresponds to $\sigma_{\mathrm{D}} / D_{\mathrm{m}}$ of only $4.6 \%$ in Eq. (12) since the changes in $K_{\mathrm{u}}$ and $D$ caused by $\sigma_{\mathrm{Ku}}$ and $\sigma_{\mathrm{D}}$ correspond to $K_{\mathrm{u}}=K_{\mathrm{um}}-\sigma_{\mathrm{Ku}}$ and $D^{2}=\left(D_{\mathrm{m}}-\sigma_{\mathrm{D}}\right)^{2}$ in Eq. (12), respectively. Therefore, even for the same numerical values of $\sigma_{\mathrm{Ku}} / K_{\mathrm{um}}$ and $\sigma_{\mathrm{D}} / D_{\mathrm{m}}, \sigma_{\mathrm{Ku}} / K_{\mathrm{um}}$ has little apparent effect.

Next, we discuss $K_{\mathrm{um}} V_{\mathrm{m}} / k T$ for various grain numbers per bit using Fig. 4. The dependence of $K_{\text {um }} V_{\mathrm{m}} / k T$ on $\sigma_{\mathrm{D}} / D_{\mathrm{m}}$ weakens as the grain number per bit increases due to a statistical problem. If one bit contains many grains, the bit error rate becomes low since the probability is very low for a simultaneous error for more than half of the grains in one bit. For example, when there are 9 error-free grains and 7 grains with errors, the bit is error free for 16 grains/bit as shown in Fig. 5 (a). However, 2 bits are error free and 2 bits have errors when there are 4 grains/bit even with the same grain magnetization arrangement as shown in Fig. 5 (b). Thus, $K_{\mathrm{um}} V_{\mathrm{m}} / k T$ decreases as the grain number per bit increases due to a statistical problem. 


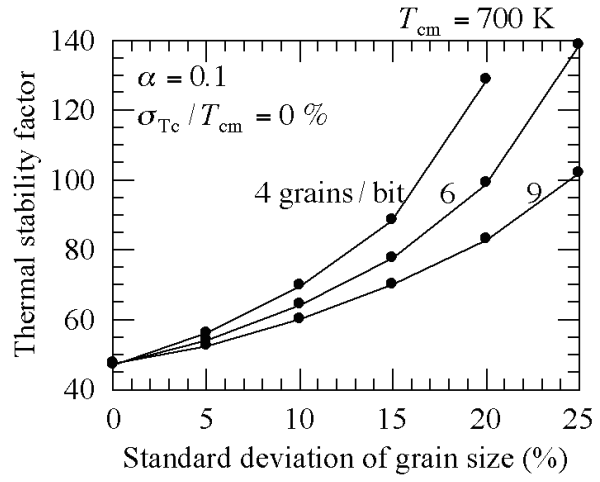

Fig. 4 Thermal stability factor necessary for 10 years of archiving as a function of the standard deviation of the grain size for various grain numbers per bit.

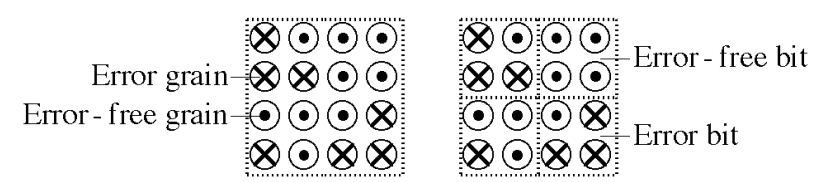

(a)

(b)
Fig. 5 (a) One bit of 16 grains/bit and (b) four bits of 4 grains/bit.

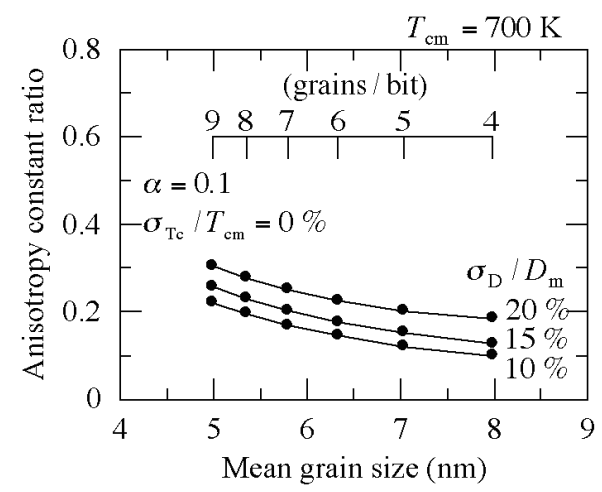

Fig. 6 Anisotropy constant ratio necessary for 10 years of archiving as a function of the mean grain size for various standard deviations of grain size $\sigma_{\mathrm{D}} / D_{\mathrm{m}}$.

Figure 6 shows $K_{\mathrm{u}} / K_{\text {bulk }}$ necessary for 10 years of archiving as a function of $D_{\mathrm{m}}$ for various $\sigma_{\mathrm{D}} / D_{\mathrm{m}}$ values. The inserted scale indicates the grain number per bit corresponding to $D_{\mathrm{m}}$ for a recording density of 2 Tbpsi. Although $K_{\mathrm{um}} V_{\mathrm{m}} / k T$ decreases as the grain number per bit increases, a larger $K_{\mathrm{u}} / K_{\text {bulk }}$ is necessary as the grain number per bit increases due to the smaller grain size.

The $K_{\mathrm{u}}$ value at a storage temperature of $300 \mathrm{~K}$ is important as regards information stability for 10 years of archiving. And $K_{\mathrm{u}}(T=300 \mathrm{~K})$ is a function of $T_{\mathrm{c}}$ as shown in Fig. 7. The $K_{\mathrm{u}}(T=300 \mathrm{~K})$ value for a medium with a low $T_{\mathrm{c}}$ is intrinsically low. Therefore, a larger $K_{\mathrm{u}} / K_{\text {bulk }}$ is necessary for a low $T_{\mathrm{c}}$ medium to obtain the same $K_{\mathrm{u}}(T=300 \mathrm{~K})$ value as that for a high $T_{\mathrm{c}}$ medium.

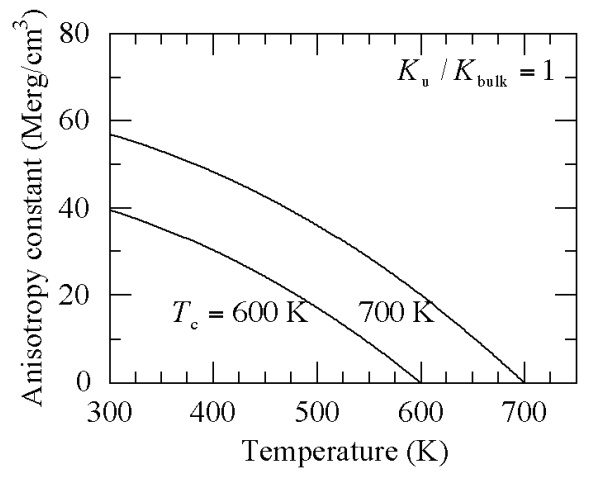

Fig. 7 Temperature dependence of anisotropy constant for Curie temperatures $T_{\mathrm{c}}=600$ and $700 \mathrm{~K}$.

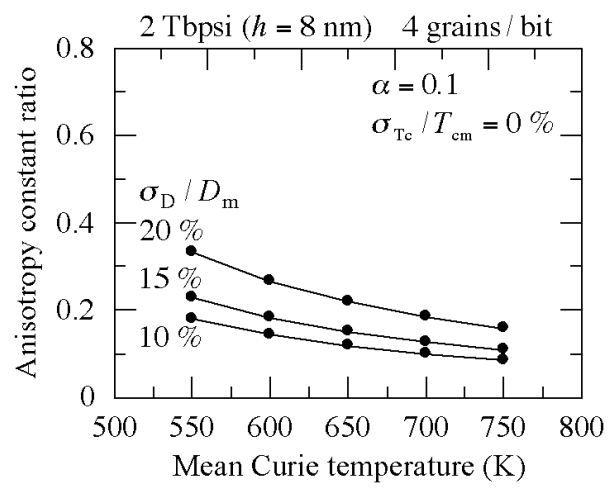

(a)

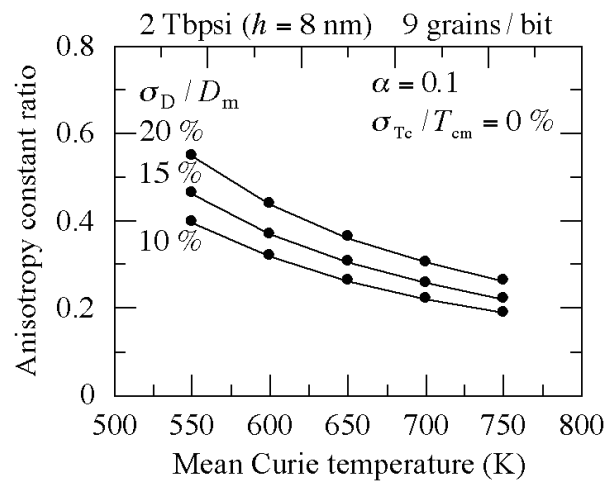

(b)

Fig. 8 Anisotropy constant ratio necessary for 10 years of archiving as a function of the mean Curie temperature for (a) 4 and (b) 9 grains/bit.

The $K_{\mathrm{u}} / K_{\text {bulk }}$ value for 10 years of archiving as a function of $T_{\mathrm{cm}}$ is shown in Fig. 8. As expected from the above discussion, a larger $K_{\mathrm{u}} / K_{\text {bulk }}$ is required as the mean Curie temperature decreases. A low Curie temperature is advantageous for writing in HAMR. However, it is disadvantageous for media manufacturing. For 9 grains/bit, as shown in Fig. 8 (b), a larger $K_{\mathrm{u}} / K_{\text {bulk }}$ is necessary than for 4 grains/bit as shown in Fig. 8 (a) due to the smaller grain size. The results are for a grain height $h$ of $8 \mathrm{~nm}$. If $h=4 \mathrm{~nm}$, namely half the height, the $K_{\mathrm{u}} / K_{\text {bulk }}$ value must be double. The required $K_{\mathrm{u}} / K_{\text {bulk }}$ value exceeds 1.0 for the combination of $2 \mathrm{Tbpsi}, 9$ grains/bit, $T_{\mathrm{cm}}=550 \mathrm{~K}$, $\sigma_{\mathrm{D}} / D_{\mathrm{m}}=20 \%$, and $h=4 \mathrm{~nm}$ even using an FePt 
medium with a large $K_{\mathrm{u}}$.

Table 2 Calculation conditions for temperature dependence of bit error rate.

\begin{tabular}{l|c} 
Recording density (Tbpsi) & 2 \\
Non - magnetic spacing $\Delta(\mathrm{nm})$ & 1 \\
Grain height $h(\mathrm{~nm})$ & 8 \\
Grain number (grains / bit) & 4 \\
Gilbert damping constant $\alpha$ & 0.1 \\
Mean Curie temperature $T_{\mathrm{cm}}(\mathrm{K})$ & 700 \\
Standard deviation of Curie temp. $\sigma_{\mathrm{Tc}} / T_{\mathrm{cm}}(\%)$ & 0 \\
Signal threshold & 0.5 \\
Bit number (bits) & $10^{7}$ \\
\hline
\end{tabular}

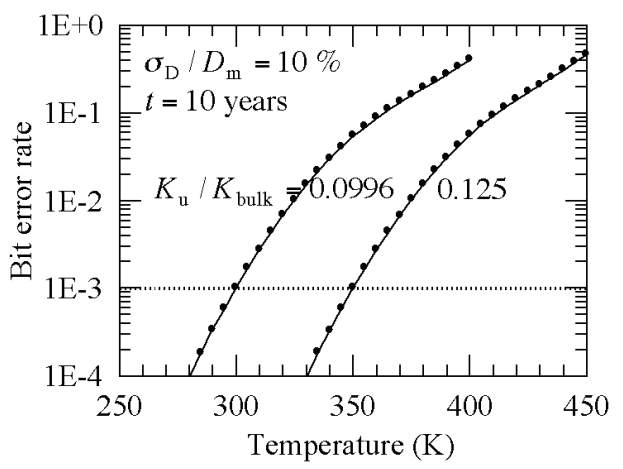

(a)

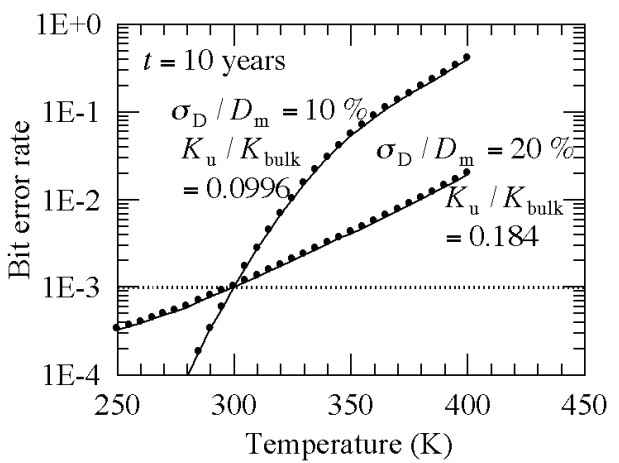

(b)

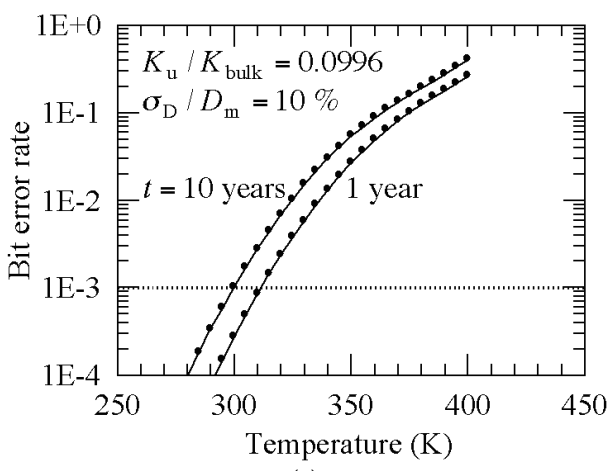

(c)

Fig. 9 Temperature dependence of bit error rate for (a) various anisotropy constant ratios $K_{\mathrm{u}} / K_{\text {bulk }}$, (b) various standard deviations of grain size $\sigma_{\mathrm{D}} / D_{\mathrm{m}}$, and (c) various times $t$.

\subsection{Temperature dependence of bit error rate}

The storage temperature is also a parameter with a strong impact since temperature is a variable of an exponential function in the grain error probability as shown in Eq. (12). Therefore, we discuss the temperature dependence of the bit error rate in this section. The calculation conditions are summarized in Table 2.

Figure 9 (a) shows the temperature dependence of the bit error rate for $K_{\mathrm{u}} / K_{\text {bulk }}=0.0996$ and 0.125 . When $K_{\mathrm{u}} / K_{\text {bulk }}=0.0996$, the bit error rate is $1 \mathrm{E}-3$ at $300 \mathrm{~K}$ for 10 years. However, the bit error rate increases rapidly as the temperature increases, and it exceeds $1 \mathrm{E}-2$ at only $325 \mathrm{~K}$. The maximum ambient temperature of a hard disk drive appears to be about $330 \mathrm{~K}$. Therefore, $K_{\mathrm{u}} / K_{\text {bulk }}=0.0996$ is insufficient. If $K_{\mathrm{u}} / K_{\text {bulk }}=0.125$, the information is stable up to $350 \mathrm{~K}$ when we take a certain margin for temperature into account.

When $\sigma_{\mathrm{D}} / D_{\mathrm{m}}$ increases from $10 \%$ to $20 \%$, the temperature dependence of the bit error rate weakens as shown in Fig. 9 (b). However, $K_{\mathrm{u}} / K_{\text {bulk }}=0.184$ is necessary for $\sigma_{\mathrm{D}} / D_{\mathrm{m}}=20 \%$ if we are to obtain the same bit error rate for $\sigma_{\mathrm{D}} / D_{\mathrm{m}}=10 \%$ at $300 \mathrm{~K}$.

The storage time $t$ is a weak impact parameter, and so the difference between the temperatures with a bit error rate of $1 \mathrm{E}-3$ for $t=1$ and 10 years is about $10 \mathrm{~K}$ as shown in Fig. 9 (c).

\section{Conclusion}

We evaluated the thermal stability factor $K_{\mathrm{um}} V_{\mathrm{m}} / k T$ and the anisotropy constant ratio $K_{\mathrm{u}} / K_{\text {bulk }}$ required for 10 years of archiving in heat-assisted magnetic recording of 2 Tbpsi employing a bit error rate calculation using the grain error probability under the conditions used in this paper. We classified the calculation parameters as those with a strong and a weak impact on the bit error rate.

The Gilbert damping constant and the Curie temperature variation are weak impact parameters. The attempt frequency can be treated as a constant.

On the other hand, the grain size variation, the grain number per bit $n$, the mean Curie temperature $T_{\mathrm{cm}}$, and the storage temperature $T$ are strong impact parameters.

The $K_{\mathrm{um}} V_{\mathrm{m}} / k T$ value is strongly dependent on the standard deviation of the grain size $\sigma_{\mathrm{D}} / D_{\mathrm{m}}$. Although $K_{\mathrm{um}} V_{\mathrm{m}} / k T$ decreases as $n$ increases due to a statistical problem, a larger $K_{\mathrm{u}} / K_{\text {bulk }}$ is necessary as $n$ increases due to a smaller grain size.

A larger $K_{\mathrm{u}} / K_{\text {bulk }}$ is also necessary as $T_{\mathrm{cm}}$ decreases. The required $K_{\mathrm{u}} / K_{\text {bulk }}$ value exceeds 1.0 for the combination of 9 grains/bit, $T_{\mathrm{cm}}=550 \mathrm{~K}, \sigma_{\mathrm{D}} / D_{\mathrm{m}}$ $=20 \%$, and a grain height of $4 \mathrm{~nm}$ even using an FePt medium.

The bit error rate increases rapidly as $T$ increases, and a certain $K_{\mathrm{u}} / K_{\text {bulk }}$ margin is required as regards temperature. 
Acknowledgement We acknowledge the support of the Advanced Storage Research Consortium (ASRC), Japan.

\section{References}

1) S. H. Charap, P. -L. Lu, and Y. He: IEEE Trans. Magn., 33, 978 (1997).

2) T. Kobayashi, T. Kitayama, and Y. Fujiwara: J. Magn. Soc. Jpn., 36, 282 (2012).

3) Y. Isowaki, T. Kobayashi, and Y. Fujiwara: J. Magn. Soc. Jpn., 38, 1 (2014).

4) E. D. Boerner and H. N. Bertram: IEEE Trans. Magn., 34, 1678 (1998).

5) T. Kobayashi, Y. Isowaki, and Y. Fujiwara: J. Magn. Soc. Jpn., 39, 8 (2015).
6) T. Kobayashi, Y. Nakatani, and Y. Fujiwara: J. Magn. Soc. Jpn., 42, 110 (2018).

7) M. Mansuripur and M. F. Ruane: IEEE Trans. Magn., MAG-22, 33 (1986).

8) J. -U. Thiele, K. R. Coffey, M. F. Toney, J. A. Hedstrom, and A. J. Kellock: J. Appl. Phys., 91, 6595 (2002).

9) Y. Nakatani, Y. Uesaka, N. Hayashi, and H. Fukushima: $J$. Magn. Magn. Mat., 168, 347 (1997).

10) D. A. Garanin and O. Chubykalo-Fesenko: Phys. Rev. B, 70, 212409 (2004).

11) T. Kobayashi, Y. Nakatani, and Y. Fujiwara: J. Magn. Soc. Jpn., 43, 70 (2019).

Received Jul. 6, 2019; Accepted Aug. 22, 2019 